T.A. Minashvili, K.D. Davitadze and I.T. Trapaidze

\title{
Effect of anisotropy of optical reflexion from the (110) surface of gallium arsenide
}

ABSTRACT. The effect of anisotropy on the optical reflexion from the surface of the (110) plane of GaAs, which is characterized by relatively low symmetry, has been investigated using modulated polarized radiation. Experiments were carried out on GaAs samples with different doping levels, as well as at a surface barrier structure made on the base of GaAs with an applied bias voltage. It was established that the effect of reflexion anisotropy, which arises in crystals with increasing charge carrier concentration and in structures with increasing bias voltage, are caused by a presurface electric field $E_{\mathrm{S}}$. Quantitative investigations showed that the amplitude of this signal increases as $E_{\mathrm{S}}{ }^{2}$.

Nanotechnology Perceptions 4 (2008) 43-46

Nonsubscribers purchase individual article 